\title{
ORIGINAL ARTICLE \\ Genetic architecture underlying morning and evening circadian phenotypes in fruit flies Drosophila melanogaster
}

\begin{abstract}
KM Vaze, KL Nikhil and VK Sharma
Circadian rhythms are perhaps among the genetically best characterized behaviours. Several mutations with drastic effects on circadian processes have been identified and models developed to explain how clock genes and their products generate self-sustained oscillations. Although natural variations in circadian phenotypes have been studied extensively, the genetic basis of such adaptive variations remains largely unknown. Here we report the results of a preliminary genetic analysis of adaptive divergence of circadian phenotypes in populations of fruit flies Drosophila melanogaster. Two sets of populations, 'early' and 'late', were created in a long-term laboratory selection for morning and evening emergence, with four independent replicates each. Over the course of $\sim 55$ generations, the early flies evolved increased morning emergence and a shorter circadian period, whereas late flies evolved increased evening emergence and longer period. To examine the genetic basis of circadian phenotypes, we set up crosses between early and late flies, and monitored emergence and activity/rest rhythms in the F1, backcrossed and F2 progeny. Our analysis suggests that the genetic basis of divergent circadian phenotypes in early and late stocks is primarily autosomal. Line-cross analysis revealed that additive and non-additive genetic effects contribute to the divergence of circadian phenotypes in early and late flies.
\end{abstract}

Heredity (2013) 111, 265-274; doi:10.1038/hdy.2013.28; published online 24 April 2013

Keywords: Drosophila; circadian; adult emergence; activity/rest; genetic bases

\section{INTRODUCTION}

Organisms ranging from bacteria to humans exhibit daily rhythms in various behavioural and physiological processes. Many of these rhythms are regulated by endogenous time-keeping systems known as circadian clocks (Roenneberg et al., 2003; Helfrich-Förster et al., 2011), which is evident from the persistence of these rhythms under constant conditions with near 24-h period. Circadian rhythms synchronize with earthly 24 -h environmental cycles, using time-cues (zeitgebers) such as cyclic light, and temperature, and attain a stable phase relationship $(\psi)$ with the environmental cycles, through a process known as 'entrainment'. Light is one of the principal entraining zeitgebers, and $\psi$ of the entrained rhythms is known to be a function of circadian period $(\tau)$ and photic phase response curve (PRC) (Johnson et al., 2003; Roenneberg et al., 2003). Circadian rhythms are one of the best understood physiological processes at the genetic and molecular levels. Genetic analyses of mutagen-induced variation in $\psi, \tau$ and PRC, have proven to be the most successful strategy in identifying genes involved in the regulation of: (1) core circadian oscillators, (2) input pathways by which environmental time cues such as light and temperature are sensed and (3) output mechanisms by which biological functions are regulated in a rhythmic manner (Bell-Pedersen et al., 2005; Hardin, 2011).

Circadian rhythms are believed to be an evolutionary adaptation to rhythmic challenges posed by daily environmental cycles and are thought to confer advantage to living beings by scheduling their behaviours and physiological processes at favourable time of the daily cycle, and by coordinating their internal physiology
(Hut and Beersma, 2011; Vaze and Sharma, 2013). Although, every point on the earth's surface experiences 24-h rhythms of various abiotic factors, natural habitats differ in terms of many features of daily cycles such as daylength, and levels of light and temperature. Such variation in features of daily environmental cycles from one geographical location to another determines the extent of rhythmic challenges and consequently the variation in rhythmic selection pressure. Therefore, genetic variation in clock properties such as $\psi$, $\tau$ and PRC among the natural populations, and their correlation with features of daily environmental cycles has been taken as evidence to suggest adaptive value of circadian clocks (Lankinen, 1986; Pittendrigh and Takamura, 1989; Costa et al., 1992; Sawyer et al., 1997; Michael et al., 2003; Tauber et al., 2007; Rieger et al., 2012). Similarly, evolution of clock properties in response to laboratory selection for timing of rhythmic behaviours provides another line of evidence for adaptive value of circadian clocks (Pittendrigh and Minis, 1971; Kumar et al., 2007; Kannan et al., 2012; Yadav and Sharma, 2013).

According to quantitative genetic theory, phenotypic variation in any trait results from alleles segregating at multiple loci (Falconer and Mackay, 1996; Hamilton, 2009), primarily through inter- and intralocus allelic interactions, such as (i) additive effects of alleles (at the same locus and across multiple loci), (ii) interaction between alleles at the same locus (dominance) and (iii) interaction among alleles at two or more loci (epistasis; Falconer and Mackay, 1996; Hamilton, 2009). Apart from the genes possessed by individuals, genotypes of interacting individuals are also known to influence the phenotype 
(Mutic and Wolf, 2007). A classic example of such effects is the influence of maternal genotype on the offspring phenotype through factors other than genes contributed by the mother, which is known as maternal genetic effects (Mousseau et al., 2009). Therefore, knowledge of genetic loci, intra locus and inter locus interactions (additive, dominance and epistatic genetic effects), and maternal effects are essential for a comprehensive understanding of the genetic architecture underlying any trait (Falconer and Mackay, 1996). Furthermore, understanding of the genetic architecture of any trait is incomplete without the knowledge of genetic basis of naturally occurring trait variation. It is well established that the contribution of non-additive genetic effects (like epistasis) can greatly complicate efforts to identify and map genes underlying complex traits (Wade, 2001). Therefore, estimates of contribution of non-additive effects to phenotypic variation may help in choosing strategies to find and map genes, and in interpreting results from quantitative trait locus analysis. Candidate gene studies based on genes identified through classical mutagenesis approach (Costa et al., 1992; Tauber et al., 2007), and quantitative trait locus analysis of circadian phenotypes (Michael et al., 2003; Kim et al., 2007), have helped in identifying the genetic basis of naturally occurring variation in circadian phenotypes. Although these approaches have been successful in the identification of genetic loci, the contributions of additive and non-additive genetic effects arising from inter- and intra-loci allelic interactions and that of maternal effects to adaptive variation in circadian phenotype are largely unknown. Except studies on mice (Shimomura et al., 2001), pitcher-plant mosquitoes Wyeomyia smithii (Mathias et al., 2006) and bean beetle Callosobruchus chinensis (Harano and Miyatake, 2010), there have hardly been any studies that throw light on the relative contribution of dominance, epistasis and maternal effects to the variation in circadian phenotypes.

Fruit flies Drosophila melanogaster exhibit robust daily rhythms in the emergence of adults from the pupal case and in its adult activity/ rest behaviour. Circadian control of these rhythms is well known and these rhythms are among the best understood circadian rhythms at the genetic, molecular and neuronal levels (Allada and Chung, 2010). Rhythm in adult emergence is observed in populations of developing individuals and activity/rest rhythm in individual adults. Under laboratory, 12-h Light/12-h Dark cycles (LD) emergence primarily occurs during the light phase (day). Each day emergence begins with lights-on, peaks during the first $2 \mathrm{~h}$ and decreases with progression of day, with the end of emergence by evening. Individual flies are also active primarily during the light phase, with peaks of activity during lights-on and lights-off (Allada and Chung, 2010). Here, we report the results of our study where we examined the genetic architecture underlying the circadian phenotypes of early and late populations of fruit flies $D$. melanogaster, created in a long-term laboratory selection study by imposing selection for adult emergence during morning and evening hours, respectively (Kumar et al., 2007). The change in trait value in response to laboratory selection is considered equivalent to evolution occurring in nature, and therefore as per convention (Garland and Rose, 2009) we have used the word 'evolution' to describe selection-mediated changes in traits. The early and late stocks evolved enhanced emergence during morning and evening hours, as a result of evolution of divergent adult emergence waveforms. Circadian clocks of early and late stocks also evolved, which was evident from divergence of $\tau$ (based on adult emergence and activity/rest rhythms) and PRC of emergence rhythm (Kumar et al., 2007). Although the phase of entrained rhythms is known to be a function of properties of circadian oscillator such as $\tau$ and PRC, they could also be a function of clock components downstream of the oscillator (Johnson et al.,
2003). Therefore, correlation between emergence timings and clock properties ( $\tau$ and PRC) in early and late stocks might not provide a proof of causal relationship. Such correlation can certainly be taken as suggestive evidence for the role of circadian clocks in the regulation of morning and evening emergence. Having characterized the circadian phenotypes, the aim of our current study was to examine the genetic architecture of circadian phenotypes of early and late populations.

In order to study the genetic bases of circadian phenotypes of early and late stocks, we set crosses between early and late stocks and obtained F1, F2 and back-crossed progeny. We scored morning and evening emergence, and estimated $\tau$ of activity rhythm as a proxy for circadian phenotypes in the progeny from 16 different types of crosses (Figure 1). This scheme of crosses allowed us to test the contributions of sex chromosomes and two types of cytoplasmic factors. Offspring receives maternal cytoplasm in the form of egg cytoplasm, which in insects is known to influence several pre-adult and adult traits (de Belle and Sokolowski, 1987). Short-lived maternal cytoplasmic factors such as hormones, messenger RNAs and proteins are known as transient maternal factors (TMF), whereas maternally inherited non-chromosomal genetic factors such as mitochondria persist

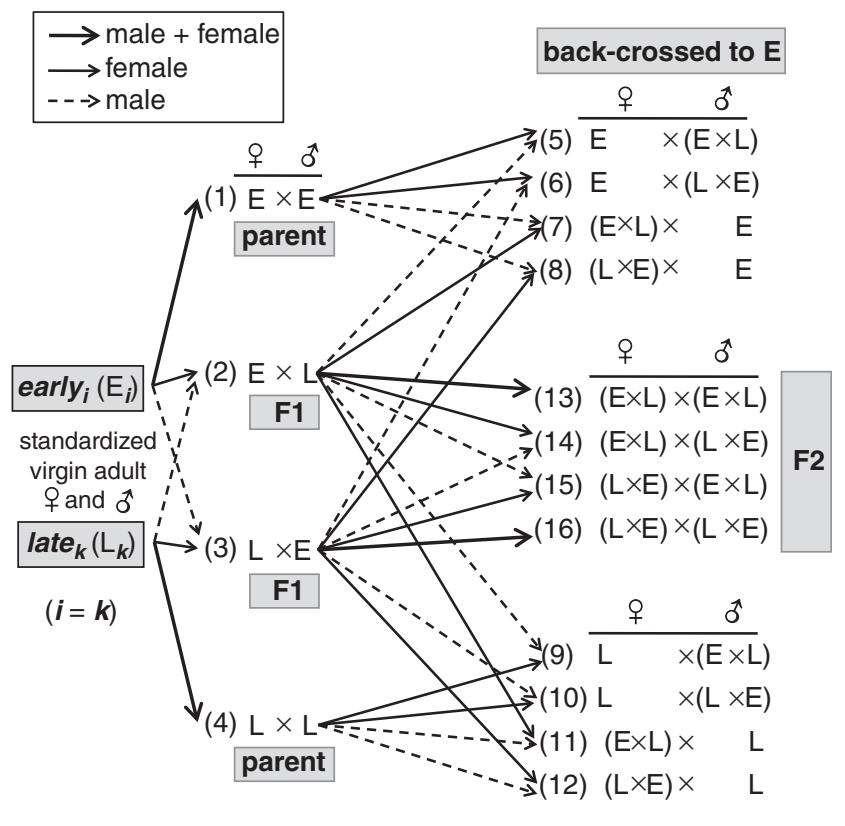

back-crossed to $\mathrm{L}$

Figure 1 Schematic diagram shows a set of crosses and the sequence in which they were performed while doing the genetic analysis of early and late circadian phenotypes. A total of 16 crosses were set up in a sequential manner in two stages. All the crosses were set up by mixing 200 virgin males and 200 virgin females of an average age of 6 days in plexiglass cages with BJ food and water ad libitum and were kept in LD conditions. In the first stage, standardized virgin males and females from a pair of early and late $_{k}$ stocks sharing the same subscript $(i=k)$ were used to set up four crosses, parental $E(1)$, parental $L(4)$, and two types of F1 progeny (2), (3). Progeny flies emerging throughout the day for 2-3 consecutive days were collected to set up crosses for the next stage. Virgin males and females of (1), (2), (3) and (4) were used to set up 12 crosses in the second stage. Reciprocal crosses between parental $E$ (1) and two types of F1 progeny (2), (3) produced four types of backcross to E progeny-(5), (6), (7) and (8). Similarly, reciprocal crosses between parental $L(4)$ and two $F 1 s$ (2), (3) yielded four types of backcross to L progeny-(9), (10), (11) and (12). Full set of reciprocal crosses between (2) and (3) produced four types of F2 progeny-(13), (14), (15) and (16). 
throughout the lifetime, and are therefore known as permanent cytoplasmic factors (PCF; de Belle and Sokolowski, 1987). Our study revealed that genetic basis of timing of emergence and $\tau$ of activity/ rest rhythm in early and late stocks is primarily autosomal. In addition, we performed line-cross analyses to examine the relative contribution of additive, non-additive (dominance + epistasis) and maternal effects to the circadian phenotypes of early and late flies. The results revealed that complex genetic architecture comprising dominance and epistasis underlie circadian phenotypes of early and late flies.

\section{MATERIALS AND METHODS}

Fly population maintenance and laboratory selection protocol

Fruit fly D. melanogaster populations selected for morning (early) and evening (late) emergence (Kumar et al., 2007) were used for the genetic analysis of morning and evening circadian phenotypes in the current study. Four replicate population, each of early $_{i=1 \ldots 4}, \operatorname{control}_{j=1 \ldots 4}$ and late $k_{k=1 \ldots 4}$ flies, were initiated from four large, outbred ancestral populations $\left(J B_{1 \ldots 4}\right)$. Each of the early, control, and late stocks sharing the same subscript were initiated from a common ancestral $J B$ population, and therefore each pair of early $y_{i}$ and late $k_{k}$ (where, $i=k$ ) populations was treated as an independent replicate. Although maintenance and selection protocol of early and late stocks are described in great detail in Kumar et al. (2007) briefly, all populations were maintained as independent units on a 21-day generation cycle, with no gene flow between them. Each fly population was maintained as a large group of adult individuals, in a plexiglass cage of $25 \times 20 \times 15-\mathrm{cm}^{3}$ dimension, with banana-jaggery food and water provided ad libitum. At every generation, eggs were collected from the cage populations ( $\sim 300$ eggs per glass vial with $6-\mathrm{ml}$ banana-jaggery food), and exactly 24, 16 and 48 such vials were set up early, control and late stocks, respectively. Throughout the pre-adult development and as adults, all populations were reared in cubicles maintained at constant temperature of $25 \pm 0.5^{\circ} \mathrm{C}$ (mean \pm s.d.) and relative humidity of $75 \pm 5 \%$. Alternating LD cycles were created with sharp dark-to-light and light-to-dark transitions, with light intensity of $\sim 0.15 \mathrm{Wm}^{-2}$ during the light phase. Time of the day under laboratory LD cycles was denoted as zeitgeber time (ZT) in hours. In every LD cycle, lights-on was considered as ZT00 and lights-off as ZT12. At every generation, populations of adults for early stocks were chosen by collecting flies that emerged during a span of $4 \mathrm{~h}$ in the morning (from $3 \mathrm{~h}$ before lights-on to $1 \mathrm{~h}$ after lights-on: ZT21-01) for 5 successive days (9th and 13th day after egg collection). Similarly, populations of adults for late stocks were chosen from flies that emerged during 4-h span in the evening ( $3 \mathrm{~h}$ before lights-off to $1 \mathrm{~h}$ after lights-off: ZT09-13). Four replicate control stocks were also maintained exactly similar to the selected populations, except that populations of control stocks were chosen from flies that emerged throughout the day, for the same 5 days. Therefore, control stocks were not under any conscious selection for timing of emergence. At the end, care was taken to ensure that each selected and control populations consisted of $\sim 1200$ individuals, with approximately equal number of males and females.

It has been observed that non-genetic factors such as altered physiology of the parents due to environmental conditions that they experience can influence the phenotype of their progeny. Consequently, selection regimes imposed on populations can also influence the phenotype of their progeny because of some unknown non-genetic effects. Therefore, we subjected selected and control populations to a common rearing condition comprising relaxed selection for one generation before conducting any assay on these populations. Hereafter, the progeny of such flies will be referred as 'standardized flies'.

\section{Crosses between early and late flies}

A total of 16 crosses were set up in a sequential manner as shown in Figure 1 for every pair of early $j_{i}$ and late $e_{k}$ (where $i=k$ ) stocks. All the analyses performed on progeny phenotypes are presented separately for each replicate pair of early and late stocks, and four replicates were named as R1, R2, R3 and R4, respectively.

\section{Adult emergence assay}

Approximately 300 eggs per vial were collected from each crossed populations, and after egg collection all vials were stored under laboratory LD conditions. After flies started to emerge, its numbers from each vial was recorded as separate counts of males and females four times a day at ZT02, 08, 14 and 20, as an estimate of emergence during morning, mid-day, evening and night windows, for up to four consecutive days. The number of flies of any given sex that emerged in a 6-h window was normalized by the total number of flies of the same sex that emerged from the same vial in that particular cycle, with ZT20 marking the start of the cycle. Cycles with less than 8 males or females from any given vial were not considered for calculation of vial average for emergence. Therefore, the number of days of data considered for any given vial varied between 2-4 days, irrespective of the population (early/late). All the vial means for percentage emergence values were arc-sine square-root transformed before doing any further analysis.

\section{Activity/rest assay}

Activity/rest behaviour of about 48 unmated male and 48 unmated female flies, collected over 2-3 consecutive days from each cross, were recorded under constant darkness (DD) for over 10 days using Drosophila Activity Monitors from Trikinetics (Waltham, MA, USA). Activity of individual flies was recorded inside locomotor activity tubes (glass tube of 5-cm length and 4-mm inner diameter) provided with standard corn-sugar-yeast medium. The $\tau$ of activity/rest rhythm was calculated for individual flies by analyzing activity data collected in 5-min bin for a minimum of 10 days, using Lomb-Scargle Periodogram in CLOCKLAB (Actimetrics, Wilmette, IL, USA).

\section{Statistical analyses}

Percentage emergence during morning or evening hours and $\tau$ of activity/rest rhythm was calculated to quantify the circadian phenotypes. Differences among the circadian phenotypes of selected and control populations were tested by carrying out mixed model analysis of variance (ANOVA). Comparisons of adult emergence data among selected and control populations was analysed by mixed model two-way ANOVA, in which selection regime (early/control/late; S), window of emergence (morning/evening; W) were considered as fixed effect factors, and four replicate populations (block-B) were treated as random factor. Comparisons of $\tau$ of activity/rest rhythm among selected and control populations were carried out by mixed model one-way ANOVA. Post hoc multiple comparisons were carried out using Tukey's test.

The scheme of crosses between early and late stocks (as shown in Figure 1 and Supplementary Table S2A) allowed us to test the contribution of four hereditary factors-sex chromosomes (X, Y) and maternal/cytoplasmic factors to the difference between early/late circadian phenotypes following the approach adopted by de Belle and Sokolowski (1987). Supplementary Table S2A provides scheme of crosses and inheritance of four hereditary factors in the progeny flies. Comparisons among mean circadian phenotypes (morning and evening emergence, and $\tau$ of activity/rest rhythm) of appropriately chosen progeny allowed us to test the contribution of each of the four factors and their interactions to the difference between early and late phenotypes, as shown in Supplementary Table S2B, following de Belle and Sokolowski (1987). Null hypothesis of no difference among mean circadian phenotypes of 16 types of progeny from crosses between each pair of early and late stocks was tested by one-factor ANOVA, followed by test of hypotheses concerning the contribution of various hereditary factors (Supplementary Table S2B), performed by planned contrast ANOVA. Differences between progeny, which share three of the four hereditary factors, were tested for significance to assess the contribution of the fourth factor. For example, in a test for contribution of PCF, female progeny of crosses 13 and 14 have the same type of $\mathrm{X}$ chromosome, autosomes and TMF but differ only in the type of PCF. Therefore, comparison of $(13+14)$ with $(15+16)$ was used to test the contribution of PCF to the differences between early and late phenotypes (Supplementary Tables S2A and B). This analysis was performed separately on each replicate pair of early and late stocks (R1, R2, R3 and R4). 


\section{Analysis of line-crosses}

Analysis of line-cross was performed to examine the contribution of additive effects (A), dominance (D), maternal effects (M) and epistasis (E) to the difference between early and late circadian phenotypes. Analyses of each of the four replicate pairs of early $y_{i}$ and late ${ }_{k}$ stocks (where $i=k$ ) were performed separately. Analysis of each trait was done separately for males and females. Mean phenotypes and variances were calculated for progeny from each of the crosses, which will be called 'generation mean' hereafter. Generation means were analysed following the protocol described in Mather and Jinks (1982). Briefly, the analysis was aimed at identifying the genetic models that best explain the variation in observed generation means. Observed generation means were fitted to genetic models comprising different combinations of A, $\mathrm{D}, \mathrm{M}$ and $\mathrm{E}$, using weighted least-square regression to estimate parameters of the genetic models, which were used to calculate the generation means expected under respective models. Goodness-of-fit of the observed generation means with those expected from the model was examined using $\chi^{2}$ test. Significant difference between observed and expected generation means in $\chi^{2}$ test would indicate that the observed means were not in agreement with those expected from the model, which then prompted testing of higher models. Lack of significance indicates that observed means are explainable by the model being tested, and thus suggest that genetic effects incorporated in the model are sufficient to explain the variation among generation means. The parameter coefficients used while estimating model parameters by weighted least-square regression are given in Supplementary Table S4. Assignment of paramete coefficients was according to Tables 11.4 and 13.2 in Kearsey and Pooni (1996) as followed by Bieri and Kawecki (2003).

We tested the goodness-of-fit of models consisting up to eight composite genetic parameters-additive (a), dominance (d), and three types of diagenic epistatic interactions-additive-additive (aa), additive-dominance (ad), dominance-dominance $(\mathrm{dd})$, maternal additive effect $\left(a_{\mathrm{m}}\right)$, maternal dominance effects $\left(d_{\mathrm{m}}\right)$ and cytoplasmic effects (c). In traditional joint scaling test, model testing starts with the simplest possible model such as the one containing only additive effects, and if simpler models are found to be inadequate in explaining variation in the observed means, further genetic effects are added sequentially in the order dominance, epistasis and maternal effects, until the observed means match those expected under the model. However, the order in which the parameters are added influences the detection of effects of parameters added later, and thus the best-fit model obtained in this manner may not provide us with the most parsimonious model. Therefore, we followed another method adopted by Bieri and Kawecki (2003), wherein every parameter could either be added or removed from the model. Although more than one model could have adequately described the observed variation in the generation means, to find the most parsimonious model we used Akaike's Information Criterion (AIC), following Bieri and Kawecki (2003), which detects the best compromise between the amount of variation explained and the number of parameters used. AIC was calculated for every candidate model tested for each trait, and the model with minimum AIC was selected as the model that best explains the observed variation in generation means.

\section{RESULTS}

Divergence of emergence preference and $\tau$ in early and late stocks We used the percentage of flies emerged during morning and evening hours as a surrogate measure for circadian emergence phenotypes of early and late stocks under LD. The early stocks showed higher emergence during morning $(\sim 63 \%)$ and a lower emergence during evening hours $(\sim 3 \%)$, whereas late stocks exhibited greater emergence during evening $(\sim 35 \%)$ and a lesser emergence in morning hours $(\sim 15 \%)$, and were found to be significantly different from each other (Supplementary Figures S1A and S1C; Supplementary Table S1A).

The $\tau$ of activity/rest rhythm in early and late stocks diverged from that of controls, and were found to be significantly different from each other (Supplementary Table S1B). The early flies evolved shorter $\tau(\sim 23.45 \mathrm{~h})$, whereas late flies evolved longer $\tau(\sim 24.20 \mathrm{~h})$ compared with controls ( $\sim 23.90 \mathrm{~h}$; Supplementary Figure S1B, Supplementary Figure S1D).

\section{Hereditary factors contributing to divergence of early and late circadian phenotypes}

Contribution of four hereditary factors X, Y chromosomes, PCF and TMF to the circadian phenotypes of early and late stocks were tested following de Belle and Sokolowski (1987); Supplementary Tables S2A and $\mathrm{B}$.

\section{Adult emergence phenotypes}

ANOVA on percentage emergence during morning and evening hours (arc-sine square-root transformed) in progeny flies showed a statistically significant effect of cross in all the four replicates $(P<0.001$; Supplementary Tables S3A and B). Planned contrasts on the morning emergence data showed that in males, $\mathrm{Y}$ chromosome (in $\mathrm{R}_{1}$ ), $\mathrm{X}$ chromosome + maternal factors (in $\mathrm{R}_{3}$ ) and TMF (in $\mathrm{R}_{4}$ ) showed a statistically significant effect. In females, none of the hereditary factors showed statistically significant effect (Table 1A). In case of evening emergence, except in $\mathrm{R}_{4}$ males where significant effect of TMF was seen, tests for none of the other hereditary factors showed statistically significant effect (Table 1B). Although genetic basis of early and late emergence phenotypes appear primarily autosomal, contribution of $\mathrm{X}$ chromosome and cytoplasmic factors is evident in some replicates.

\section{$\tau$ of activity/rest rhythm}

ANOVA on the $\tau$ of activity/rest rhythm in progeny flies showed a statistically significant effect of cross in all the four replicates (Supplementary Table S3C). Planned contrast analysis revealed that in males TMF (in $\mathrm{R}_{2}$ ) and interaction of TMF with other factors (in $\mathrm{R}_{4}$ ) had statistically significant effect, whereas tests for none of the other hereditary factors showed statistically significant effect in $\mathrm{R}_{1}$ or $\mathrm{R}_{3}$ males or in any of the replicates in females (Table 1C). Therefore, genetic basis of divergent $\tau$ of activity/rest rhythm in early and late flies is primarily autosomal.

\section{Line-cross analyses}

Analysis of line-crosses between early and late flies revealed a complex genetic architecture underlying their circadian phenotypes. Genetic architecture underlying $\tau$ was relatively simpler than that of morning and evening emergence. For each trait, genetic architecture of circadian phenotypes varied among the replicates and differed between males and females. Tables 2A-C show the parameter estimates for the genetic model that best explained variations in the generation means among all the models that were tested (that is, the model with smallest AIC value).

\section{Emergence during morning hours}

Crosses between early and late flies revealed that additive, dominance and epistatic effects contribute to the differences in morning emergence (Table 2A; Figure 2a). In males, difference in morning emergence in only one out of four replicates (R2) was explainable by simple additive effects alone, but incorporation of dominance and epistasis improved the fit (Table 2A; Supplementary Table S5). In the remaining three replicates, none of the genetic effect models including dominance, maternal effects and diagenic epistasis were able to explain the difference between early and late stocks (Table 2A; Supplementary Table S5). In females, difference in morning emergence was not explainable by simple additive model in any of the four replicates (Table 2A; Supplementary Table S6). In two replicates $\left(R_{1}\right.$ and $R_{2}$ ) additive and epistatic effect model was sufficient to explain 
Table 1 Results of planned comparisons performed to test the contribution of various hereditary factors to the circadian phenotypes of early and late stocks: (A), morning emergence; (B), evening emergence; $(C)$ circadian period $(\tau)$ of activity/rest rhythm

\begin{tabular}{|c|c|c|c|c|c|c|c|c|c|}
\hline \multirow[b]{2}{*}{$\begin{array}{l}\text { (A) } \\
0\end{array}$} & & \multicolumn{2}{|c|}{$R 1$} & \multicolumn{2}{|c|}{$R 2$} & \multicolumn{2}{|c|}{$R 3$} & \multicolumn{2}{|c|}{$R 4$} \\
\hline & & $\begin{array}{l}d f \\
\alpha^{\prime}\end{array}$ & $\begin{array}{c}101 \\
0.0064\end{array}$ & $\begin{array}{l}d f \\
\alpha^{\prime}\end{array}$ & $\begin{array}{l}103 \\
0.0064\end{array}$ & $\begin{array}{l}d f \\
\alpha^{\prime}\end{array}$ & $\begin{array}{l}85 \\
0.0064\end{array}$ & $\begin{array}{l}d f \\
\alpha^{\prime}\end{array}$ & $\begin{array}{c}89 \\
0.0064\end{array}$ \\
\hline Test & & $F$ & $P$ value & $F$ & $P$ value & $F$ & $P$ value & $F$ & $P$ value \\
\hline $\begin{array}{l}1 \\
3 \\
7 \\
8 \\
9 \\
10 \\
11 \\
12\end{array}$ & $\begin{array}{l}P \\
X+M \\
Y \\
Y \text { interaction } \\
P C F \\
P C F \text { intearctions } \\
\text { TMF } \\
\text { TMF interaction }\end{array}$ & $\begin{array}{r}259.95 \\
1.46 \\
9.07 \\
5.67 \\
4.21 \\
1.05 \\
0.05 \\
0.50\end{array}$ & $\begin{array}{c}0.001^{*} \\
0.220 \\
0.003^{*} \\
0.019 \\
0.040 \\
0.300 \\
0.820 \\
0.480\end{array}$ & $\begin{array}{r}119.14 \\
1.06 \\
0.57 \\
3.12 \\
0.43 \\
0.26 \\
0.21 \\
0.82\end{array}$ & $\begin{array}{l}0.001^{*} \\
0.300 \\
0.450 \\
0.080 \\
0.510 \\
0.610 \\
0.640 \\
0.360\end{array}$ & $\begin{array}{r}109.26 \\
9.02 \\
0.02 \\
0.00 \\
2.01 \\
1.00 \\
1.96 \\
0.12\end{array}$ & $\begin{array}{l}0.001^{*} \\
0.003^{*} \\
0.880 \\
1.000 \\
0.150 \\
0.320 \\
0.160 \\
0.720\end{array}$ & $\begin{array}{r}64.40 \\
0.00 \\
1.15 \\
0.10 \\
0.06 \\
1.38 \\
13.59 \\
2.91\end{array}$ & $\begin{array}{l}0.001^{*} \\
1.00 \\
0.280 \\
0.750 \\
0.800 \\
0.240 \\
0.001 * \\
0.091\end{array}$ \\
\hline ㅇ & & $\begin{array}{l}d f \\
\alpha^{\prime}\end{array}$ & $\begin{array}{c}101 \\
0.0073\end{array}$ & $\begin{array}{l}d f \\
\alpha^{\prime}\end{array}$ & $\begin{array}{l}103 \\
0.0073\end{array}$ & $\begin{array}{l}d f \\
\alpha^{\prime}\end{array}$ & $\begin{array}{c}85 \\
0.0073\end{array}$ & $\begin{array}{l}d f \\
\alpha^{\prime}\end{array}$ & $\begin{array}{c}89 \\
0.0073\end{array}$ \\
\hline Test & & $F$ & $P$ value & $F$ & $P$ value & $F$ & $P$ value & $F$ & $P$ value \\
\hline $\begin{array}{l}1 \\
2 \\
4 \\
5 \\
6 \\
9 \\
10\end{array}$ & $\begin{array}{l}P \\
D \\
M \\
X \\
X \text { interactions } \\
P C F \\
P C F \text { interactions }\end{array}$ & $\begin{array}{r}284.17 \\
0.07 \\
2.62 \\
2.81 \\
0.21 \\
0.04 \\
0.03\end{array}$ & $\begin{array}{l}0.001^{*} \\
0.790 \\
0.100 \\
0.090 \\
0.640 \\
0.840 \\
0.860\end{array}$ & $\begin{array}{r}280.98 \\
0.07 \\
0.11 \\
0.36 \\
0.61 \\
0.03 \\
3.92\end{array}$ & $\begin{array}{l}0.001^{*} \\
0.790 \\
0.740 \\
0.540 \\
0.430 \\
0.860 \\
0.050\end{array}$ & $\begin{array}{r}192.29 \\
4.70 \\
0.07 \\
0.89 \\
0.99 \\
0.87 \\
0.05\end{array}$ & $\begin{array}{l}0.001^{*} \\
0.032 \\
0.790 \\
0.340 \\
0.320 \\
0.350 \\
0.820\end{array}$ & $\begin{array}{r}136.99 \\
0.09 \\
0.25 \\
0.26 \\
0.23 \\
0.00 \\
1.31\end{array}$ & $\begin{array}{l}0.001{ }^{*} \\
0.760 \\
0.610 \\
0.610 \\
0.630 \\
1.000 \\
0.250\end{array}$ \\
\hline $\begin{array}{l}(B) \\
0\end{array}$ & & $\begin{array}{l}d f \\
\alpha^{\prime}\end{array}$ & $\begin{array}{c}101 \\
0.0063\end{array}$ & $\begin{array}{l}d f \\
\alpha^{\prime}\end{array}$ & $\begin{array}{l}103 \\
0.0063\end{array}$ & $\begin{array}{l}d f \\
\alpha^{\prime}\end{array}$ & $\begin{array}{c}85 \\
0.0063\end{array}$ & $\begin{array}{l}d f \\
\alpha^{\prime}\end{array}$ & $\begin{array}{c}89 \\
0.0063\end{array}$ \\
\hline Test & & $F$ & $P$ value & $F$ & $P$ value & $F$ & $P$ value & $F$ & $P$ value \\
\hline $\begin{array}{l}1 \\
3 \\
7 \\
8 \\
9 \\
10 \\
11 \\
12\end{array}$ & $\begin{array}{l}P \\
X+M \\
Y \\
Y \text { interaction } \\
P C F \\
P C F \text { intearctions } \\
\text { TMF } \\
\text { TMF interaction }\end{array}$ & $\begin{array}{r}199.01 \\
0.01 \\
7.57 \\
0.59 \\
1.70 \\
0.36 \\
3.13 \\
0.68\end{array}$ & $\begin{array}{l}0.001^{*} \\
0.890 \\
0.007 \\
0.440 \\
0.190 \\
0.540 \\
0.070 \\
0.410\end{array}$ & $\begin{array}{r}174.22 \\
0.11 \\
0.03 \\
0.46 \\
0.09 \\
0.03 \\
0.13 \\
0.41\end{array}$ & $\begin{array}{l}0.001^{*} \\
0.740 \\
0.860 \\
0.490 \\
0.760 \\
0.860 \\
0.710 \\
0.520\end{array}$ & $\begin{array}{r}131.28 \\
0.84 \\
0.30 \\
0.24 \\
1.15 \\
1.37 \\
1.65 \\
3.18\end{array}$ & $\begin{array}{l}0.001^{*} \\
0.360 \\
0.580 \\
0.620 \\
0.280 \\
0.240 \\
0.200 \\
0.078\end{array}$ & $\begin{array}{r}109.68 \\
1.09 \\
0.76 \\
0.39 \\
0.83 \\
0.46 \\
17.27 \\
0.08\end{array}$ & $\begin{array}{l}0.001^{*} \\
0.290 \\
0.380 \\
0.530 \\
0.360 \\
0.410 \\
0.001^{*} \\
0.770\end{array}$ \\
\hline q & & $\begin{array}{l}d f \\
\alpha^{\prime}\end{array}$ & $\begin{array}{c}101 \\
0.0073\end{array}$ & $\begin{array}{l}d f \\
\alpha^{\prime}\end{array}$ & $\begin{array}{l}103 \\
0.0073\end{array}$ & $\begin{array}{l}d f \\
\alpha^{\prime}\end{array}$ & $\begin{array}{c}85 \\
0.0073\end{array}$ & $\begin{array}{l}d f \\
\alpha^{\prime}\end{array}$ & $\begin{array}{c}89 \\
0.0073\end{array}$ \\
\hline Test & & $F$ & $P$ value & $F$ & $P$ value & $F$ & $P$ value & $F$ & $P$ value \\
\hline $\begin{array}{l}1 \\
2 \\
4 \\
5 \\
6 \\
9 \\
10\end{array}$ & $\begin{array}{l}P \\
D \\
M \\
X \\
X \text { interactions } \\
P C F \\
P C F \text { interactions }\end{array}$ & $\begin{array}{r}10.34 \\
0.13 \\
0.73 \\
1.26 \\
1.18 \\
0.03 \\
0.04\end{array}$ & $\begin{array}{l}0.001 * \\
0.710 \\
0.390 \\
0.260 \\
0.270 \\
0.860 \\
0.840\end{array}$ & $\begin{array}{r}87.50 \\
0.04 \\
0.03 \\
0.85 \\
1.63 \\
0.00 \\
1.05\end{array}$ & $\begin{array}{l}0.001^{*} \\
0.840 \\
0.860 \\
0.350 \\
0.200 \\
1.000 \\
0.300\end{array}$ & $\begin{array}{r}156.21 \\
9.93 \\
0.07 \\
2.14 \\
0.63 \\
0.34 \\
0.41\end{array}$ & $\begin{array}{l}0.001^{*} \\
0.002^{*} \\
0.790 \\
0.140 \\
0.420 \\
0.560 \\
0.520\end{array}$ & $\begin{array}{r}146.64 \\
5.10 \\
3.52 \\
4.39 \\
2.06 \\
2.68 \\
1.95\end{array}$ & $\begin{array}{l}0.001^{*} \\
0.026 \\
0.063 \\
0.038 \\
0.150 \\
0.100 \\
0.160\end{array}$ \\
\hline $\begin{array}{l}\text { (C) } \\
0\end{array}$ & & $\begin{array}{l}d f \\
\alpha^{\prime}\end{array}$ & $\begin{array}{c}523 \\
0.0064\end{array}$ & $\begin{array}{l}d f \\
\alpha^{\prime}\end{array}$ & $\begin{array}{l}603 \\
0.0064\end{array}$ & $\begin{array}{l}d f \\
\alpha^{\prime}\end{array}$ & $\begin{array}{c}535 \\
0.0064\end{array}$ & $\begin{array}{l}d f \\
\alpha^{\prime}\end{array}$ & $\begin{array}{c}582 \\
0.0064\end{array}$ \\
\hline Test & & $F$ & $P$ value & $F$ & $P$ value & $F$ & $P$ value & $F$ & $P$ value \\
\hline $\begin{array}{l}1 \\
3 \\
7 \\
8 \\
9 \\
10 \\
11 \\
12\end{array}$ & $\begin{array}{l}P \\
X+M \\
Y \\
Y \text { interaction } \\
P C F \\
P C F \text { intearctions } \\
\text { TMF } \\
\text { TMF interaction }\end{array}$ & $\begin{array}{r}84.14 \\
4.64 \\
0.59 \\
1.08 \\
0.01 \\
2.70 \\
2.47 \\
1.23\end{array}$ & $\begin{array}{l}0.001^{*} \\
0.031 \\
0.440 \\
0.290 \\
0.310 \\
0.100 \\
0.110 \\
0.260\end{array}$ & \begin{tabular}{r|}
80.17 \\
5.78 \\
0.24 \\
2.84 \\
2.22 \\
0.45 \\
17.01 \\
4.90 \\
\end{tabular} & $\begin{array}{l}0.001^{*} \\
0.016 \\
0.620 \\
0.090 \\
0.130 \\
0.500 \\
0.001^{*} \\
0.027\end{array}$ & \begin{tabular}{r|}
66.62 \\
1.70 \\
0.50 \\
0.39 \\
0.21 \\
0.47 \\
6.06 \\
0.29
\end{tabular} & $\begin{array}{l}0.001^{*} \\
0.190 \\
0.470 \\
0.530 \\
0.640 \\
0.490 \\
0.014 \\
0.590\end{array}$ & $\begin{array}{r}42.03 \\
1.29 \\
0.36 \\
5.59 \\
0.12 \\
5.09 \\
6.21 \\
11.26\end{array}$ & $\begin{array}{l}0.001 \text { * } \\
0.260 \\
0.540 \\
0.018 \\
0.720 \\
0.024 \\
0.018 \\
0.001 *\end{array}$ \\
\hline q & & $\begin{array}{l}d f \\
\alpha^{\prime}\end{array}$ & $\begin{array}{c}521 \\
0.0073\end{array}$ & $\begin{array}{l}d f \\
\alpha^{\prime}\end{array}$ & $\begin{array}{l}481 \\
0.0073\end{array}$ & $\begin{array}{l}d f \\
\alpha^{\prime}\end{array}$ & $\begin{array}{c}467 \\
0.0073\end{array}$ & $\begin{array}{l}d f \\
\alpha^{\prime}\end{array}$ & $\begin{array}{c}464 \\
0.0073\end{array}$ \\
\hline Test & & $F$ & $P$ value & $F$ & $P$ value & $F$ & $P$ value & $F$ & $P$ value \\
\hline $\begin{array}{l}1 \\
2 \\
4 \\
5 \\
6 \\
9 \\
10\end{array}$ & $\begin{array}{l}\mathrm{P} \\
\mathrm{D} \\
\mathrm{M} \\
\mathrm{X} \\
\mathrm{X} \text { interactions } \\
\mathrm{PCF} \\
\mathrm{PCF} \text { interactions }\end{array}$ & $\begin{array}{r}48.43 \\
2.34 \\
0.00 \\
0.69 \\
0.00 \\
1.47 \\
0.58\end{array}$ & $\begin{array}{l}0.001 * \\
0.120 \\
1.000 \\
0.400 \\
1.000 \\
0.220 \\
0.440\end{array}$ & $\begin{array}{r}33.22 \\
0.04 \\
5.13 \\
3.31 \\
0.59 \\
3.11 \\
0.99\end{array}$ & $\begin{array}{l}0.001 * \\
0.840 \\
0.020 \\
0.060 \\
0.440 \\
0.070 \\
0.320\end{array}$ & $\begin{array}{r}34.80 \\
2.39 \\
0.01 \\
0.08 \\
1.15 \\
0.72 \\
2.28\end{array}$ & $\begin{array}{l}0.001^{*} \\
0.120 \\
0.920 \\
0.770 \\
0.280 \\
0.390 \\
0.130\end{array}$ & $\begin{array}{r}18.32 \\
0.53 \\
0.02 \\
0.01 \\
0.20 \\
0.01 \\
0.00\end{array}$ & $\begin{array}{l}0.001 \text { * } \\
0.460 \\
0.880 \\
0.920 \\
0.650 \\
0.920 \\
1.000\end{array}$ \\
\hline
\end{tabular}

Abbreviations: $\alpha^{\prime}$, per comparison error rate to keep experiment-wise error rate at $\alpha=0.05$, calculated according to Dunn-Sidak method; D, dominance; X,Y, X/Y chromosome; df, denominator degrees of freedom (numerator df is 1 for all comparisons); M, maternal effects (PCF + TMF); P, difference between parents; PCF, permanent cytoplasmic factors; TMF, transient maternal factors. Significant comparisons are indicated by '*'. 
Table 2 Estimates of model parameters, $\chi^{2}$ values for the model, which best explained the variation in emergence phenotypes and in period of activity/rest rhythm in the progeny from crosses between early and late stocks: (A), morning emergence; (B), evening emergence; $(C)$, circadian period $(\tau)$ of activity/rest rhythm

\begin{tabular}{|c|c|c|c|c|}
\hline Parameters & $R 1$ & $R 2$ & $R 3$ & $R 4$ \\
\hline \multicolumn{5}{|l|}{$\begin{array}{l}\text { (A) } \\
\text { Male }\end{array}$} \\
\hline $\begin{array}{l}\text { m } \\
\text { a } \\
\text { d } \\
\text { aa } \\
\text { ad } \\
\text { dd }\end{array}$ & $\begin{array}{l}38.95 \pm 0.04 \\
14.22 \pm 1.04\end{array}$ & $\begin{array}{c}29.01 \pm 2.99 \\
10.92 \pm 0.96 \\
22.64 \pm 7.8 \\
8.73 \pm 2.83 \\
-13.1 \pm 5.27\end{array}$ & $\begin{array}{c}44.21 \pm 1.1 \\
12.84 \pm 1.86 \\
-4.95 \pm 2.06 \\
-9.85 \pm 4.34 \\
-8.81 \pm 3.22\end{array}$ & $\begin{array}{c}39.34 \pm 0.53 \\
13.2 \pm 1.32\end{array}$ \\
\hline $\begin{array}{l}\chi^{2} \\
\mathrm{df} \\
P \\
\text { AIC }\end{array}$ & $\begin{array}{l}32.37 \\
14 \\
0.003 \\
S \\
36.37\end{array}$ & $\begin{array}{l}11.58 \\
11 \\
0.396 \\
\text { NS } \\
21.58\end{array}$ & $\begin{array}{c}21.75 \\
11 \\
0.026 \\
S \\
31.76\end{array}$ & $\begin{array}{c}36.05 \\
14 \\
0.001 \\
S \\
40.06\end{array}$ \\
\hline $\begin{array}{l}\text { Female } \\
\text { m } \\
\text { a } \\
\text { d } \\
\text { aa } \\
\text { ad } \\
\text { dd }\end{array}$ & $\begin{array}{c}42.39 \pm 2.48 \\
18.74 \pm 0.8 \\
-23.8 \pm 5.58 \\
-7.35 \pm 1.79 \\
24.77 \pm 3.61\end{array}$ & $\begin{array}{l}41.09 \pm 0.84 \\
15.25 \pm 1.27 \\
-5.99 \pm 1.65 \\
-6.47 \pm 1.79\end{array}$ & $\begin{array}{l}38.69 \pm 0.48 \\
12.91 \pm 1.54\end{array}$ & $\begin{array}{c}36.44 \pm 0.42 \\
15.13 \pm 1.2\end{array}$ \\
\hline $\begin{array}{l}\chi^{2} \\
\mathrm{df} \\
P \\
\text { AIC }\end{array}$ & $\begin{array}{l}11.17 \\
11 \\
0.429 \\
\text { NS } \\
21.17\end{array}$ & $\begin{array}{c}16.16 \\
12 \\
0.180 \\
\text { NS } \\
24.17\end{array}$ & $\begin{array}{c}54.8 \\
14 \\
0.000 \\
S \\
58.8\end{array}$ & $\begin{array}{c}24.66 \\
14 \\
0.038 \\
5 \\
28.66\end{array}$ \\
\hline \multicolumn{5}{|l|}{$\begin{array}{l}\text { (B) } \\
\text { Male }\end{array}$} \\
\hline $\begin{array}{l}\mathrm{m} \\
\mathrm{a} \\
\mathrm{d}\end{array}$ & $\begin{array}{l}22.75 \pm 0.55 \\
16.25 \pm 1.12\end{array}$ & $\begin{array}{c}20.36 \pm 1.1 \\
14.78 \pm 1.11\end{array}$ & $\begin{array}{l}27.75 \pm 1.23 \\
11.86 \pm 0.86 \\
-6.09 \pm 2.29\end{array}$ & $\begin{array}{l}30.05 \pm 0.97 \\
14.28 \pm 1.89\end{array}$ \\
\hline $\begin{array}{l}\text { aa } \\
\text { dd }\end{array}$ & & $\begin{array}{l}5.04 \pm 1.61 \\
4.91 \pm 1.34\end{array}$ & & $\begin{array}{c}-5.92 \pm 2.19 \\
-5.4 \pm 1.71\end{array}$ \\
\hline $\begin{array}{l}\chi^{2} \\
\mathrm{df} \\
P \\
\text { AIC }\end{array}$ & $\begin{array}{l}56.53 \\
14 \\
0.000 \\
S \\
60.54\end{array}$ & $\begin{array}{c}7.82 \\
12 \\
0.799 \\
\text { NS } \\
15.83\end{array}$ & $\begin{array}{l}17.07 \\
13 \\
0.195 \\
\text { NS } \\
23.08\end{array}$ & $\begin{array}{c}22.85 \\
12 \\
0.029 \\
S \\
30.85\end{array}$ \\
\hline $\begin{array}{l}\text { Female } \\
\text { m } \\
\text { a } \\
\text { aa } \\
\text { ad } \\
\text { dd }\end{array}$ & $\begin{array}{c}28.79 \pm 0.65 \\
13.14 \pm 0.78 \\
-10.4 \pm 1.16 \\
4.72 \pm 1.98 \\
-10.3 \pm 0.93\end{array}$ & $\begin{array}{l}19.25 \pm 0.8 \\
17.52 \pm 1.2 \\
4.49 \pm 1.52 \\
5.8 \pm 1.52\end{array}$ & $\begin{array}{c}28.89 \pm 1.15 \\
18.11 \pm 1.4 \\
-4.62 \pm 1.88 \\
-9.42 \pm 3.15\end{array}$ & $\begin{array}{c}27.09 \pm 0.57 \\
15.94 \pm 1.4\end{array}$ \\
\hline $\begin{array}{l}\chi^{2} \\
\mathrm{df} \\
P \\
\text { AIC }\end{array}$ & $\begin{array}{c}6.04 \\
11 \\
0.870 \\
\text { NS } \\
16.05\end{array}$ & $\begin{array}{c}23.69 \\
12 \\
0.020 \\
S \\
31.7\end{array}$ & $\begin{array}{l}15.68 \\
12 \\
0.206 \\
\text { NS } \\
23.68\end{array}$ & $\begin{array}{c}45.45 \\
14 \\
0.000 \\
S \\
49.45\end{array}$ \\
\hline $\begin{array}{l}\text { (C) } \\
\text { Male }\end{array}$ & & & & \\
\hline $\begin{array}{l}\mathrm{m} \\
\mathrm{a} \\
a_{\mathrm{m}}\end{array}$ & $\begin{array}{c}23.68 \pm 0.033 \\
0.33 \pm 0.047 \\
0.11 \pm 0.042\end{array}$ & $\begin{array}{l}23.79 \pm 0.03 \\
0.43 \pm 0.063\end{array}$ & $\begin{array}{c}23.92 \pm 0.021 \\
0.37 \pm 0.04 \\
0.1 \pm 0.048\end{array}$ & $\begin{array}{c}23.92 \pm 0.025 \\
0.34 \pm 0.048\end{array}$ \\
\hline $\begin{array}{l}\chi^{2} \\
\mathrm{df} \\
P \\
\text { AIC }\end{array}$ & $\begin{array}{c}9.6 \\
13 \\
0.725 \\
\text { NS } \\
15.61\end{array}$ & $\begin{array}{c}45.4 \\
14 \\
0.000 \\
\text { S } \\
49.4\end{array}$ & $\begin{array}{c}13.19 \\
13 \\
0.433 \\
\text { NS } \\
19.19\end{array}$ & $\begin{array}{c}24.96 \\
14 \\
0.034 \\
S \\
28.96\end{array}$ \\
\hline $\begin{array}{l}\text { Female } \\
\text { m } \\
\text { a } \\
\text { aa }\end{array}$ & $\begin{array}{c}23.97 \pm 0.036 \\
0.32 \pm 0.037 \\
-0.13 \pm 0.055\end{array}$ & $\begin{array}{c}23.92 \pm 0.022 \\
0.25 \pm 0.045\end{array}$ & $\begin{array}{l}23.99 \pm 0.024 \\
0.322 \pm 0.043\end{array}$ & $\begin{array}{c}23.99 \pm 0.016 \\
0.3 \pm 0.032\end{array}$ \\
\hline $\begin{array}{l}\chi^{2} \\
\mathrm{df} \\
P \\
\text { AIC }\end{array}$ & $\begin{array}{c}9.35 \\
13 \\
0.746 \\
\text { NS } \\
15.36\end{array}$ & $\begin{array}{c}32.49 \\
14 \\
0.003 \\
\mathrm{~S} \\
36.5\end{array}$ & $\begin{array}{c}25.91 \\
14 \\
0.026 \\
\mathrm{~S} \\
29.91\end{array}$ & $\begin{array}{c}13.36 \\
14 \\
0.498 \\
\text { NS } \\
17.37\end{array}$ \\
\hline
\end{tabular}

Abbreviations: a, additive effect; aa, additive-additive interaction; ad, additive-dominance interaction; $a_{\mathrm{m}}$, additive maternal effect; $\mathrm{AIC}=$ Akaike information criteria value (as described in Bieri and Kawecki (2003); c, cytoplasmic factors; d, dominance; dd, dominance-dominance interaction; $\mathrm{df}$, degrees of freedom used for testing goodness-of-fit $(\mathrm{df}=16-\mathrm{k}) ; d_{\mathrm{m}}$, dominance maternal effect; $\mathrm{k}$, number of significant parameters; m, mean; $\mathrm{S}$, significant $\gamma^{2}$ test.

R1, R2, R3, and R4 indicate replicate populations. Values in upper parts of columns R1, R2, $\mathrm{R} 3$, and R4 are estimates of model parameters used to calculate expected generation means. Significant $\chi^{2}$ test means model is inadequate to explain variation. the differences, but in R1, addition of dominance effects improved the fit (Table 2A; Supplementary Table S6). In two other replicates $\left(\mathrm{R}_{3}\right.$ and $\mathrm{R}_{4}$ ), none of the models could explain the differences (Table 2A; Supplementary Table S6). Together, these results suggest the role of additive and non-additive (diagenic epistatic and higher-order interactions) genetic factors in the regulation of morning emergence in early and late populations.

\section{Emergence during evening hours}

Like the genetic architecture of morning emergence, additive and epistatic interactions were required to adequately explain the differences in evening emergence between early and late stocks (Table 2B; Figure 2b). In males, additive-dominance model was sufficient to explain the differences in evening emergence in one of the replicates $\left(R_{3}\right)$, whereas in $\left(R_{2}\right)$ the differences were explainable by additive and diagenic epistatic effect model. In case of two replicates $\left(R_{1}\right.$ and $\left.R_{4}\right)$, none of the models were sufficient to explain the differences between early and late flies (Table 2B; Supplementary Table S7). In females, additive and diagenic epistasis model adequately explained the differences in evening emergence in two of the four replicates $\left(R_{1}\right.$ and $\left.R_{3}\right)$, whereas in the other two replicates $\left(R_{2}\right.$ and $\left.R_{4}\right)$, none of the models were sufficient to explain the differences in evening emergence (Table 2B; Supplementary Table S8). These results suggest a role of additive, dominance and epistatic interactions in the regulation of evening emergence.

\section{$\tau$ of activity/rest rhythm}

Crosses between early and late flies revealed that genetic architecture underlying $\tau$ of activity rhythm is relatively simpler than that underlying timing of emergence (Table 2C; Figure 2c). Difference in $\tau$ of two replicates each in male $\left(R_{1}\right.$ and $\left.R_{3}\right)$ and female $\left(R_{1}\right.$ and $\left.R_{4}\right)$ was explainable by simple additive model (Table 2C; Supplementary Tables S9 and S10). In case of males ( $\mathrm{R}_{1}$ and $\left.\mathrm{R} 3\right)$, fit of the observed generation means was better after the addition of maternal effects (Table 2C; Supplementary Table S9), whereas in R1 females addition of epistatic effects improved the fit (Table 2C; Supplementary Table S10). Differences in $\tau$ in both males and females of the remaining replicates could not be explained by any of the higher-order models (Table 2C). These results suggest that non-additive genetic interactions and maternal effects underlie divergent $\tau$ of activity/rest rhythm in early and late populations.

\section{DISCUSSION}

\section{Contribution of sex chromosomes and maternal factors}

Our analyses revealed that morning, and evening emergence, and $\tau$ of activity/rest rhythm did not differ between males of the reciprocal F1 crosses (Test 3, Tables 1A-C; Supplementary Table S2B; except for morning emergence in $\mathrm{R}_{3}$ males), which suggests that $\mathrm{X}$ chromosome does not contribute to the differences between the circadian phenotypes of males in early and late stocks. In females also, effect of $\mathrm{X}$ chromosome did not reach the level of statistical significance (Test 5, Tables 1A-C; Supplementary Table S2B). The three circadian phenotypes of F2 males (Test 7, Tables 1A-C; Supplementary Table S2B) were statistically not different (except for morning emergence in R1 males, Table 1A), suggesting lack of contribution of $\mathrm{Y}$ chromosome to the differences in circadian phenotypes of early and late stocks. Moreover, contribution of interaction of $\mathrm{X}$ chromosome with other hereditary factors (in females, Test 6, Tables 1A-C; Supplementary Table S2B) and of Y chromosome with other hereditary factors (Test 8, Tables 1A-C; Supplementary Table S2B) did not reach level of statistical significance in case of any of the circadian phenotypes. 
a
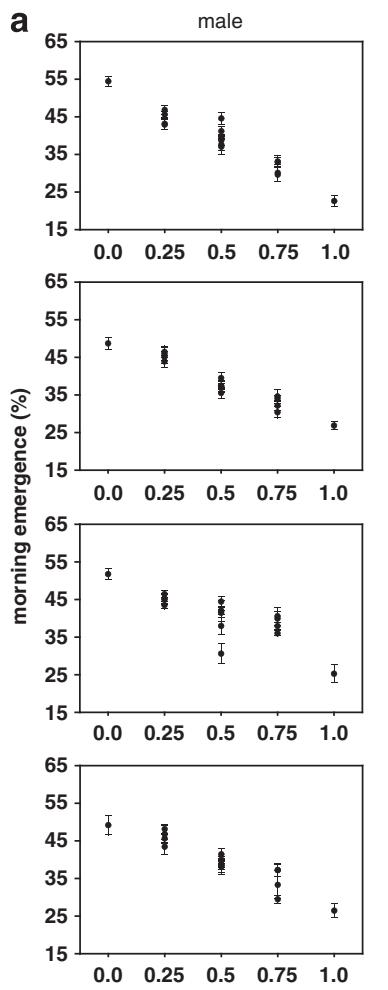

proportionc
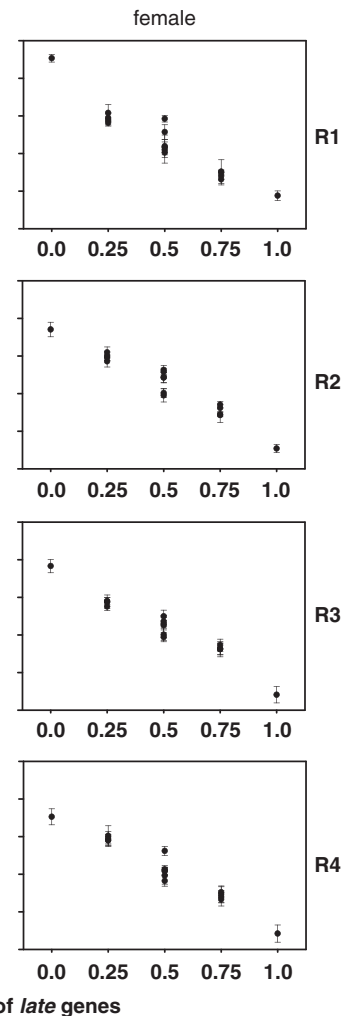

C
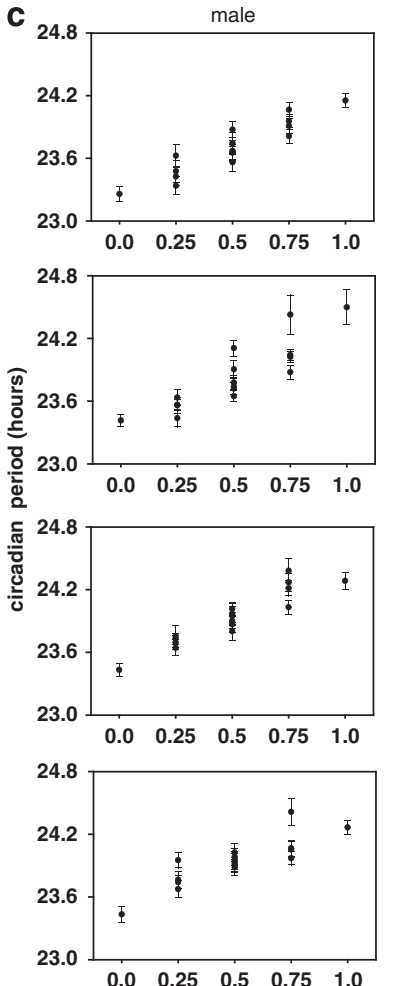

b
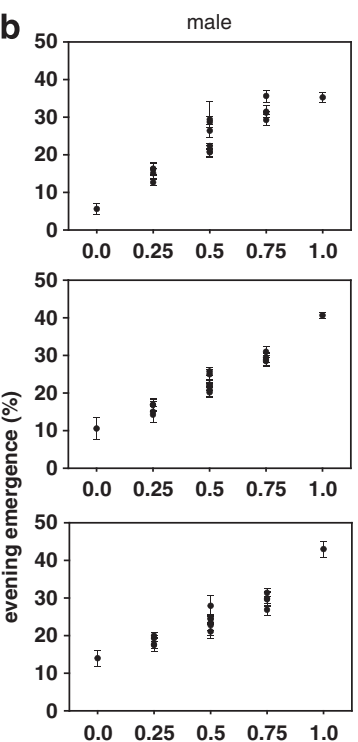

$\begin{array}{llllll}0.0 & 0.25 & 0.5 & 0.75 & 1.0\end{array}$

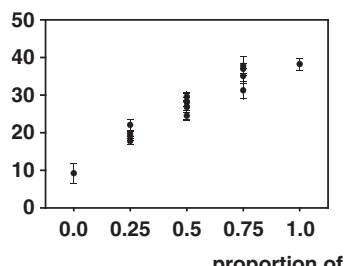

proportion of late genes
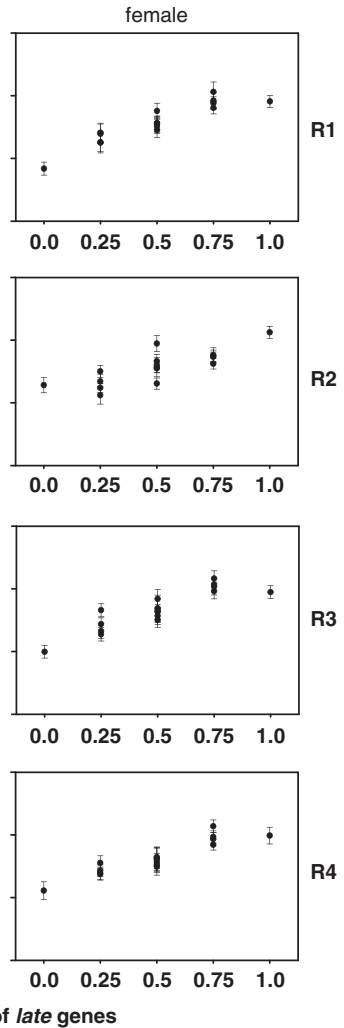

Figure 2 Circadian phenotypes in early-late parental stocks and in their F1, F2 and backcrossed progeny. Genotypes of parental stocks (early and late) and progeny from their crosses (F1, F2 and backcross) are expressed in units of the proportion of late genes (for example, in F1 and F2 progeny, 50\% of the genome is from late and so they are represented by 0.5). R1, R2, R3 and R4 indicate replicate populations. Error bars around mean circadian phenotypes are s.e.m. (a) Morning emergence in early-late parental stocks and in their F1, F2 and backcross progeny. Morning emergence was calculated as mean percentage emergence during 6-h span from ZT20-02 in replicate vials. (b) Evening emergence in early-late parental stocks and in their F1, F2 and backcross progeny. Evening emergence was calculated as mean percentage emergence during 6-h span from ZT08-14 in replicate vials. (c) $\tau$ of activity/rest rhythm in early-late parental stocks and in their F1, F2 and backcrossed progeny. 
Together, these results suggest that sex chromosomes do not contribute to the divergence of circadian phenotypes in early and late stocks.

Analyses of contribution of PCF and its interactions with other hereditary factors revealed that they too do not contribute to the differences between the circadian phenotypes of early and late stocks (Tests 9, 10, Tables 1A-C; Supplementary Table S2B). Contribution of TMF and its interactions with other hereditary factors were testable only in males (Tests 11, 12, Tables 1A-C; Supplementary Table S2B). Out of four replicate populations, in $\mathrm{R}_{4}$ alone, TMF showed a significant effect on morning and evening emergence; however, interaction of TMF with all other factors showed a statistically significant effect on $\tau$ (Tables 1A-C), indicating that maternal effects do not contribute to the differences between the circadian phenotypes of early and late stocks. Furthermore, absence of any effect of PCF suggests that non-chromosomal genetic factors (mitochondria) do not contribute to the differences in the circadian phenotypes of early and late stocks. From these results it appears that on an average, the genetic basis underlying circadian phenotypes of early and late stocks is primarily autosomal and there is no contribution of maternal effects.

The lack of contribution of $\mathrm{X}$ chromosome to the circadian phenotypes of early and late stocks may seem anomalous in light of the fact that $\mathrm{X}$ chromosome constitutes almost one-third of the total genome in D. melanogaster, and at least two known core clock genes period and shaggy are located on it (Hardin, 2011). Our results thus suggest that $\mathrm{X}$-linked clock genes are not involved in causing divergence in circadian phenotypes between early and late stocks, probably because of the lack of segregating allelic variation for $\mathrm{X}$-linked clock genes in our baseline populations.

\section{Line-cross analysis}

Line-cross analysis is by far the most powerful method to detect contribution of non-additive genetic effects to the divergence in mean phenotype among two populations or species (Demuth and Wade, 2006). Our analyses revealed that in at least half of the replicates, variation among generation means (for all the three traits) was not adequately explained by any of the models tested (Tables 2A-C; Figures $2 \mathrm{a}-\mathrm{c}$ ). Failure of any of the models to explain variation in case of several replicates could be due to the limitation of diagenic epistasis models to detect gene interactions involving more than two loci (Teotonio et al., 2004), which indicates the contribution of linkage and/or higher-order interactions to the variation in those replicates. In addition, the Mather and Jinks method (Kearsey and Pooni, 1996) assumes that the parent populations are inbred and isogenic, and therefore another reason for the lack-of-fit may be the presence of multiple alleles. Despite the inadequacy of any of the tested models to explain trait variation among generation means in those replicates, additive effects were highly significant (Tables $2 \mathrm{~A}-\mathrm{C}$ ), and pattern of generation means was nearly linear in each of those replicates (Figures $2 \mathrm{a}-\mathrm{c})$. This indicates that most variation in the circadian phenotypes of early and late stocks is explainable by additive genetic effects. Thus, the higher-order interactions and/or linkage, which is possibly the reason for the inadequacy of all the tested models to explain the variations, and is probably relatively small but significant contributor. Therefore, estimates of broad sense and narrow sense heritability would provide a greater insight into the relative contribution of additive and non-additive genetic effects to the trait variation. However, heritability estimates (obtained by the analysis of generation variances as per the method described in Kearsey and Pooni (1996); Lynch and Walsh (1998) for all the three traits (morning, evening emergence and $\tau$ of activity/rest rhythm), revealed that in many replicates the estimates were biologically meaningless $(<0$ or $>1)$, probably owing to very high s.e. of variance estimates (heritability estimates, Supplementary Table S11; variance estimates of parent, F1, backcross and F2 generations, Supplementary Figures S2A-C). Therefore, heritability estimates could not be used to judge the relative contribution of additive and non-additive effects to the divergence of circadian phenotypes between early and late stocks. Our evidence thus far suggests that although non-additive effects were essential to explain variation, substantial portion of the variation was explainable by additive effects.

Our analysis showed that no single pattern of additive, nonadditive and maternal effects was sufficient to explain divergent circadian phenotypes of early and late stocks for all the replicates. Different patterns of contribution of additive, non-additive and maternal effects among the four replicates suggest that distinct genetic architecture underlie divergent circadian phenotypes of early/late stocks. However, as discussed earlier, given the indication of additive effects as a primary cause of variation, observed differences in genetic architectures among replicate populations are although real, may be of less importance. This aspect of our results is similar to the observations in other studies. Crosses between populations of fruit flies D. pseudoobscura maintained at different temperatures indicated the evolution of different patterns of epistatic interactions among replicate populations (Mourad, 1965; Mathias et al., 2006) also reported differences in genetic architecture underlying divergent critical photoperiod and circadian phenotypes among replicate populations of pitcher-plant mosquito W. smithii selected for different critical photoperiod. Observed differences in genetic architecture underlying divergent circadian phenotypes among replicate early and late populations in our study may be attributable to inherent genetic variation among replicate populations possibly because of: (1) the replicate baseline populations in our study (from which each replicate early and late populations were initiated) may have differed genetically due to 'founder effect' (Falconer and Mackay, 1996) experienced by rare alleles in the ancestral population while founding of the replicate populations; (2) after the founding of replicate populations, they were maintained as independent populations for $\sim 700$ generations before the initiation of selection for morning and evening emergence (Kumar et al., 2007). Consequently, mutational histories experienced by the individual replicates during this period may have further caused genetic divergence among them (Teotonio et al., 2004), and (3) the variation in effective selection pressures among replicate early/late populations could be an additional source of genetic variation. Therefore, different patterns of additive and nonadditive effects to the divergent circadian phenotypes among replicate early and late populations could be attributable to (1) inherent genetic variation for circadian phenotypes among replicate populations, which is independent of genetic change brought about by selection for morning/evening emergence, and (2) an interaction between inherent genetic differences among founding populations and genetic change brought about by imposed selection.

Large outbred populations under divergent selection are thought to undergo phenotypic differentiation through selection on additive genetic variation (Coyne et al., 1997). Consistent with this notion, divergence in several fitness traits between populations of fruit flies D. melanogaster, selected for increased starvation resistance, or for shorter lifespan, has been found to be primarily contributed by additively acting genes (Teotonio et al., 2004). However, contribution of non-additive genetic effects observed in house fly populations selected for morphological characters and for courtship behaviour 
(Meffert, 2000), suggests that contribution of such non-additive genetic effects to the divergence of phenotypes in large populations is likely.

\section{Epistasis and its implications}

Our analyses revealed evidence for the contribution of non-additive genetic effects such as dominance and epistasis to the adult emergence waveforms and the $\tau$ of activity/rest rhythm in early and late stocks. Apart from it being a description of the genetic architecture underlying the circadian phenotypes of early and late stocks, evidence for contribution of epistasis to the variation in circadian phenotypes may have deep implications for molecular level model building of circadian clocks. The contribution of epistasis indicates that the phenotypic effects of an allele at a particular locus are dependent upon alleles present at other loci (Demuth and Wade, 2006). Forward genetic screens have been the workhorse in the identification of genes involved in circadian processes, and over the years several gene mutations showing effects on circadian rhythms have been identified in systems spanning bacteria to mammals (Bell-Pedersen et al., 2005; Hardin, 2011). Subsequent studies on the behaviour of products (messenger RNA, proteins) of such mutant and wild-type alleles in the presence and absence of mutant alleles at other loci have led to the development of molecular models to explain how clock genes and their products generate self-sustained oscillations, sense environmental time cues and regulate timing of target behaviours/ physiological processes (Bell-Pedersen et al., 2005; Hardin, 2011). However, these models have been typically based on the molecular analyses of inbred strains, which lack or have limited allelic variation at any given locus (for exmple, wild-type Canton-S strain of D. melanogaster), and may not necessarily hold true if tested on variable genetic backgrounds, especially for genes with subtle effects on circadian phenotypes, considering the evidence for epistasis (Rutila et al., 1996; Shimomura et al., 2001). If this is the case then the validity of such models in natural populations, which harbour much of allelic variation, may need to be reconsidered. Taken together, this suggests the need for a cautious approach towards defining the roles of genes in the so-called circadian clockwork without the knowledge of phenotypic effects of mutations on different genetic backgrounds.

\section{Differences in genetic architectures of emergence and activity/rest rhythms}

We found that the genetic architectures differed between emergence behaviour and $\tau$ of activity/rest rhythm. First of all, adult emergence and activity are two different behaviours and they were measured under two different environmental conditions, LD (entrained) and DD (free-running), respectively. Therefore, the observed differences may primarily be due to different sets of genes associated with the two behaviours, environmental effects, or due to differential gene and environment interaction. In light of the standard input-oscillatoroutput model of clocks (Allada and Chung, 2010), $\tau$ (DD phenotype) only provides information about oscillator, whereas entrained behaviour (LD phenotype) includes contribution of all the components. Therefore, differences in genetic architectures between emergence and $\tau$ are likely to be due to genes coding for input and output components in addition to oscillator components.

\section{Summary}

In summary, the results of our study suggest that divergence of early and late circadian phenotypes is primarily attributable to additive and non-additive genetic effects of autosomal loci. Also, distinct patterns of contribution of additive, non-additive effects and maternal effects to the early and late circadian phenotypes among replicates suggest multiple genetic solutions to the evolution of similar circadian phenotypes under uniform selection pressures. This knowledge may prove valuable while further elucidating the genetic architecture underlying divergent circadian phenotypes of early and late stocks. Having found that genetic basis of circadian phenotypes in early and late populations is primarily autosomal, the next logical step would be to use multiple complementary approaches, such as chromosome substitution analysis and whole genome sequencing to identify the putative genetic loci. Subsequently, these loci can be examined for their causal role in the regulation of circadian phenotypes of early and late flies.

\section{DATA ARCHIVING}

Data deposited in the Dryad repository: doi:10.5061/dryad.4c77r.

\section{CONFLICT OF INTEREST}

The authors declare no conflict of interest.

\section{ACKNOWLEDGEMENTS}

We thank Nisha, Pankaj, Joydeep, Shankari and Xavier for their assistance during population maintenance and assays. We are thankful to Sheeba Vasu and NV Joshi for helpful discussions and suggestions for improvement of the manuscript. We also thank three anonymous reviewers for suggesting several useful changes. KMV thanks CSIR, India for research fellowship.

Allada R, Chung BY (2010). Circadian organization of behavior and physiology in Drosophila. Annu Rev Physiol 72: 605-624.

Bell-Pedersen D, Cassone VM, Earnest DJ, Golden SS, Hardin PE, Thomas TL et al. (2005). Circadian rhythms from multiple oscillators: lessons from diverse organisms. Nat Rev Genet 6: 544-556.

Bieri J, Kawecki TJ (2003). Genetic architecture of differences between populations of cowpea weevil (Callosobruchus maculatus) evolved in the same environment. Evolution 57: 274-287.

Costa R, Peixoto AA, Barbujani G, Kyriacou CP (1992). A latitudinal cline in a Drosophila clock gene. Proc $R$ Soc $B$ 250: 43-49.

Coyne JA, Barton NH, Turelli M (1997). Perspective: a critique of Sewall Wright's shifting balance theory of evolution. Evolution 51: 643-671.

de Belle JS, Sokolowski MB (1987). Heredity of rover/sitter: alternative foraging strategies of Drosophila melanogaster larvae. Heredity 59: 73-83.

Demuth JP, Wade MJ (2006). Experimental methods for measuring gene interactions. Annu Rev Ecol Evol Syst 37: 289-316.

Falconer DS, Mackay TFC (1996). Introduction to Quantitative Genetics, 4th edn. Longmans Green: Harlow, Essex, UK.

Garland T, Rose MR (2009). Experimental Evolution: Concepts, Methods, and Applications of Selection Experiments. University of California Press: Berkeley, California, USA.

Hamilton M (2009). Population Genetics. Wiley-Blackwell: New Jersey, USA.

Harano T, Miyatake T (2010). Genetic basis of incidence and period length of circadian rhythm for locomotor activity in populations of a seed beetle. Heredity 105: 268-273.

Hardin T (2011). Molecular genetic analysis of circadian timekeeping in Drosophila. Adv Genet 74: 141-173.

Helfrich-Förster C, Nitabach MN, Holmes TC (2011). Insect circadian clock outputs. Essays Biochem 49: 87-101.

Hut RA, Beersma DG (2011). Evolution of time-keeping mechanisms: early emergence and adaptation to photoperiod. Philos Trans $R$ Soc London B Biol Sci 366 : 2141-2154.

Johnson CH, Elliott JA, Foster RG (2003). Entrainment of circadian programs. Chronobiol Int 20: 741-774.

Kannan NN, Vaze KM, Sharma VK (2012). Clock accuracy and precision evolve as a consequence of selection for adult emergence in a narrow window of time in fruit flies Drosophila melanogaster. J Exp Biol 215: 3527-3534.

Kearsey MJ, Pooni HS (1996). The Genetical Analysis of Quantitative Traits. Stanley Thornes: Cheltenham, UK.

Kim TS, Logsdon BA, Park S, Mezey JG, Lee K (2007). Quantitative trait loci for the circadian clock in Neurospora crassa. Genetics 177: 2335-2347.

Kumar S, Kumar D, Paranjpe DA, Akarsh CR, Sharma VK (2007). Selection on the timing of adult emergence results in altered circadian clocks in fruit flies Drosophila melanogaster. J Exp Biol 210: 906-918. 
Lankinen P (1986). Geographical variation in circadian eclosion rhythm and photoperiodic adult diapause in Drosophila littoralis. J Comp Physiol A 159: 123-142.

Lynch M, Walsh B (1998). Genetics and Analysis of Quantitative Traits. Sinauer Associates, Inc.: Sunderland, Massachusetts, USA.

Mather K, Jinks JL (1982). Biometrical Genetics: The Study of Continuous Variation. Cambridge University Press: Cambridge, UK.

Mathias D, Reed LK, Bradshaw WE, Holzapfel CM (2006). Evolutionary divergence of circadian and photoperiodic phenotypes in the pitcher-plant mosquito, Wyeomyia smithii. J Biol Rhythms 21: 132-139.

Meffert LM (2000). The evolutionary potential of morphology and mating behaviour. In Brodie ED III, Wade MJ (eds) Epistasis and the Evolutionary Process. Oxford University Press: Oxford, UK.

Michael TP, Salome PA, Yu HJ, Spencer TR, Sharp EL, McPeek MA et al. (2003). Enhanced fitness conferred by naturally occurring variation in the circadian clock. Science 302: 1049-1053.

Mourad F (1965). Genetic divergence in M. Vetukhiv's experimental populations of Drosophila pseudoobscura. Genet Res 6: 139-146.

Mousseau TA, Uller T, Wapstra E, Badyaev AV (2009). Evolution of maternal effects: past and present. Philos Trans R Soc Lond B Biol Sci 364: 1035-1038.

Mutic JJ, Wolf JB (2007). Indirect genetic effects from ecological interactions in Arabidopsis thaliana. Mol Ecol 16: 2371-2381.

Pittendrigh CS, Minis DH (1971). The photoperiodic time measurement in Pectinophora gossypiella and its relation to the circadian system in that species. In: Menaker M (ed.) Biochronometry. National Academy of Sciences: Washington DC, pp 212-250.

Pittendrigh CS, Takamura T (1989). Latitudinal clines in the properties of a circadian pacemaker. J Biol Rhythms 4: 217-235.
Rieger D, Peschel N, Dusik V, Glotz S, Helfrich-Förster C (2012). The ability to entrain to long photoperiods differs between 3 Drosophila melanogaster wild-type strains and is modified by twilight simulation. J Biol Rhythms 27: 37-47.

Roenneberg T, Daan S, Merrow M (2003). The art of entrainment. J Biol Rhythms 18: 183-194.

Rutila JE, Zeng H, Le M, Curtin KD, Hall JC, Rosbash M (1996). The tim ${ }^{S L}$ mutant of the Drosophila rhythm gene timeless manifests allele-specific interactions with period gene mutants. Neuron 17: 921-999.

Sawyer LA, Hennessy JM, Peixoto AA, Rosato E, Parkinson H, Costa R et al. (1997). Natural variation in a Drosophila clock gene and temperature compensation. Science 278: 2117-2120.

Shimomura K, Low-Zeddies SS, King DP, Steeves TD, Whiteley A, Kushla J et al. (2001). Genome-wide epistatic interaction analysis reveals complex genetic determinants of circadian behavior in mice. Genome Res 11: 959-980.

Tauber E, Zordan M, Sandrelli F, Pegoraro M, Osterwalder N, Breda C et al. (2007). Natura selection favors a newly derived timeless allele in Drosophila melanogaster. Science 316: 1895-1898.

Teotonio H, Matos M, Rose MR (2004). Quantitative genetics of functional characters in Drosophila melanogaster populations subjected to laboratory selection. J Genet 83: 265-277.

Vaze KM, Sharma VK (2013). On the adaptive significance of circadian clocks for their owners. Chronobiol Int 30: 413-433.

Wade MJ (2001). Epistasis, complex traits, and mapping genes. Genetica 112-113: 59-69.

Yadav P, Sharma VK (2013). Correlated changes in circadian clocks in response to selection for faster pre-adult development in fruit flies Drosophila melanogaster. $J$ Comp Physiol B 183: 333-343.

Supplementary Information accompanies this paper on Heredity website (http://www.nature.com/hdy) 